\title{
The threshold of stochastic chemostat model with Monod-Haldane response function
}

Zhongwei Cao ${ }^{\mathrm{a}}$, Liya Liu ${ }^{\mathrm{b}, *}$

${ }^{a}$ Department of Applied Mathematics, Jilin University of Finance and Economics, Changchun 130117, P. R. China.

${ }^{b}$ College of Science, China University of Petroleum, Qingdao 266580, Shandong Province, P. R. China.

Communicated by A. Atangana

\begin{abstract}
This paper deals with problem of a stochastic chemostat model with Monod-Haldane response function. Firstly, we confirm the truth of the existence and uniqueness of the positive solution to the system. Then, we show the condition for the microorganism to be extinct. Moreover, we investigate there is a stationary distribution of this stochastic system and finally, we derive the expression for its invariant density. (C)2017 All rights reserved.
\end{abstract}

Keywords: Stochastic chemostat model, threshold, extinction, persistence, stationary distribution. 2010 MSC: 60H10, 93E03, 93E15.

\section{Introduction}

The chemostat plays an important role in mathematical biology and theoretical ecology. A chemostat is a bioreactor to which fresh medium is continuously added, while culture liquid containing left over nutrients, metabolic end products and microorganisms are continuously removed at the same rate to keep the culture volume constant $[4,17]$. The theoretical investigation was initiated by Monod [16], Novick and Szilard [17]. In the simple case, the chemostat with single species and single substrate was proposed in [3].

In recent years, the Monod-Haldane type response function has been studied. A chemostat model with Monod-Haldane response function can be expressed as the following equations:

$$
\left\{\begin{array}{l}
\frac{d S(t)}{d t}=D\left(S^{0}-S(t)\right)-\frac{m S(t) x(t)}{a+S(t)+K S(t)^{2}}, \\
\frac{d x(t)}{d t}=\left[\frac{m S(t)}{a+S(t)+K S(t)^{2}}-D\right] x(t),
\end{array}\right.
$$

where $S(t), x(t)$ stand for the concentrations of the nutrient and the microorganism at time $t$ respectively. $S^{0}$ and D are positive constants, which respectively represent the original concentration of nutrient and

\footnotetext{
*Corresponding author

Email addresses: Caozw963@sina.com (Zhongwei Cao), liuliya_1993@hotmail.com (Liya Liu)
} 
the common washout rate. $\frac{m S(t) x(t)}{a+S(t)+K S(t)^{2}}$ denotes the Monod-Haldane growth functional response, where $m>0$ is the maximal growth rate and $a>0$ is called the Michaelis-Menten constant. Assume the term $\mathrm{KS}(\mathrm{t})^{2}$ as an inhibitor and $\mathrm{K}$ is a half-saturation parameter.

System (1.1) has a trivial equilibrium point $\left(S^{0}, 0\right)$, it is a steady node when $R_{0}:=\frac{m S^{0}}{a+S^{0}+K\left(S^{0}\right)^{2}}<D$ and a saddle point when $R_{0}>D$. What is more, there are two interior equilibrium $\left(S_{1}^{*}, x_{1}^{*}\right),\left(S_{2}^{*}, x_{2}^{*}\right)$ where $\mathrm{S}_{1}^{*}, \mathrm{~S}_{2}^{*}$ satisfy the equation

$$
\mathrm{DKS}^{2}+(\mathrm{D}-\mathrm{m}) \mathrm{S}+\mathrm{Da}=0, \quad \mathrm{D}<\mathrm{m} .
$$

In addition, one of the two interior equilibriums is a steady nodal point and the other is a saddle point. When the orbits tend to the steady nodal point, the microorganism is persistent. A certain amount of microbial is removed from the chemostat constantly. For more details, we can refer to [2].

While, inevitably, the ecosystem dynamics is affected by environmental white noise which is an important component in real applications [7-13, 19]. We cannot ignore the difference that may happen. In model (1.1), all parameters are affected by environmental noise and they always fluctuate around some average values. In this paper, we only consider the case that the maximal growth rate $m$, which is one of the crucial parameters to the continuous culture of microorganism, is perturbed by environmental white noise with

$$
m \rightarrow m+\sigma \dot{B}(t),
$$

where $B(t)$ is for standard Brownian motion on the complete probability space, $\sigma^{2}>0$ represents the intensity of the white noise. Then the stochastic model is as follows

$$
\left\{\begin{array}{l}
d S(t)=\left[D\left(S^{0}-S(t)\right)-\frac{m S(t) x(t)}{a+S(t)+K S(t)^{2}}\right] d t-\frac{\sigma S(t) x(t)}{a+S(t)+K S(t)^{2}} d B(t), \\
d x(t)=\left[\frac{m S(t)}{a+S(t)+K S(t)^{2}}-D\right] x(t) d t+\frac{\sigma S(t) x(t)}{a+S(t)+K S(t)^{2}} d B(t) .
\end{array}\right.
$$

Since the model has a positive invariant set $\left\{(S, x) \in \mathbb{R}_{+}^{2}: S+x=S^{0}\right\}$, we only have to study the equation:

$$
d x=\left[\frac{m\left(S^{0}-x\right)}{a+\left(S^{0}-x\right)+K\left(S^{0}-x\right)^{2}}-D\right] x d t+\frac{\sigma\left(S^{0}-x\right) x}{a+\left(S^{0}-x\right)+K\left(S^{0}-x\right)^{2}} d B_{t},
$$

with the initial value $x(0)=x_{0} \in\left(0, S^{0}\right)$. In this paper, we will focus on the dynamical behavior of system (1.3).

Throughout this paper, let $(\Omega, \mathscr{F}, \mathrm{P})$ be a complete probability space with a filtration $\left\{\mathscr{F}_{\mathrm{t}}\right\}_{\mathrm{t} \geqslant 0}$ satisfying the usual conditions (i.e., it is increasing and right continuous while $\mathscr{F}_{0}$ contains all $P$-null sets) and $\mathrm{B}(\mathrm{t})$ be a scalar Brownian motion defined on the probability space.

In general, consider the $d$-dimensional stochastic differential equation

$$
d x(t)=f(x(t), t) d t+g(x(t), t) d B(t), \quad \text { for } t \geqslant t_{0},
$$

with initial value $x(0)=x_{0} \in \mathbb{R}^{\mathrm{d}}$. $\mathrm{B}(\mathrm{t})$ denotes an $\mathrm{n}$-dimensional standard Brownian motion defined on the complete probability space $\left(\Omega, \mathscr{F},\left\{\mathscr{F}_{\mathrm{t}}\right\}_{\mathrm{t} \geqslant 0}, \mathrm{P}\right)$. Denote by $\mathrm{C}^{2,1}\left(\mathbb{R}^{\mathrm{d}} \times\left[\mathrm{t}_{0}, \infty\right] ; \mathbb{R}_{+}\right)$the family of all nonnegative functions $V(x, t)$ defined on $\mathbb{R}^{\mathrm{d}} \times\left[\mathrm{t}_{0}, \infty\right]$ such that they are continuously twice differentiable in $x$ and once in $t$. The differential operator $L$ of (1.4) is defined by [15]

$$
L=\frac{\partial}{\partial t}+\sum_{i=1}^{d} f_{i}(x, t) \frac{\partial}{\partial x_{i}}+\frac{1}{2} \sum_{i, j=1}^{d}\left[g^{\top}(x, t) g(x, t)\right]_{i j} \frac{\partial^{2}}{\partial x_{i} \partial x_{j}} .
$$

If $L$ acts on a function $V \in C^{2,1}\left(\mathbb{R}^{\mathrm{d}} \times\left[\mathrm{t}_{0}, \infty\right] ; \mathbb{R}_{+}\right)$, then

$$
L V(x, t)=V_{t}(x, t)+V_{x}(x, t) f(x, t)+\frac{1}{2} \operatorname{trace}\left[g^{\top}(x, t) V_{x x}(x, t) g(x, t)\right],
$$

where $V_{t}=\frac{\partial V}{\partial t}, V_{x}=\left(\frac{\partial V}{\partial x_{1}}, \ldots, \frac{\partial V}{\partial x_{d}}\right), V_{x x}=\left(\frac{\partial^{2} V}{\partial x_{i} \partial x_{j}}\right)_{d \times d}$. By Itô's formula [15, 18], if $x(t) \in \mathbb{R}^{d}$, then

$$
d V(x(t), t)=L V(x(t), t) d t+V_{x}(x(t), t) g(x(t), t) d B(t) .
$$




\section{Existence and uniqueness of the global positive solution}

To investigate the dynamics of stochastic chemostat model (1.3), the existence of a global positive solution is required first of all. And if the system (1.2) has a unique global solution, so does the system (1.3). Therefore, in the following, we will only need to show there is a unique global positive solution to (1.2). However, the coefficients of (1.2) do not satisfy the linear growth condition, though coefficients of (1.2) are locally Lipschitz continuous. As a result, the system only has a unique positive local solution for any given initial value. The following theorem tells us this solution is global.

Theorem 2.1. For any initial value $\left(S_{0}, x_{0}\right) \in \mathbb{R}_{+}^{2}$, there is a unique solution $(S(t), x(t))$ of system (1.2) on $t \geqslant 0$, and the solution will remain in $\mathbb{R}_{+}^{2}$ with probability one, namely, $(\mathrm{S}(\mathrm{t}), \mathrm{x}(\mathrm{t})) \in \mathbb{R}_{+}^{2}$ for all $\mathrm{t} \geqslant 0$ almost surely.

Proof. Since the coefficients of (1.2) are locally Lipschitz continuous, for any given initial value $\left(S_{0}, x_{0}\right) \in$ $\mathbb{R}_{+}^{2}$, there exists a unique positive local solution $(S(t), x(t))$ on $t \in\left[0, \tau_{e}\right)$ a.s., where $\tau_{e}$ is the explosion time $[1,15]$. In order to prove this solution is global, it is sufficient to show $\tau_{e}=\infty$ almost surely.

Let $n_{0}>0$ be sufficiently large such that $S(0), x(0)$ all lie within the interval $\left[\frac{1}{n_{0}}, n_{0}\right]$. For any integer $n \geqslant n_{0}$, define the stopping time:

$$
\tau_{n}=\inf \left\{t \in\left[0, \tau_{e}\right): \min \{S(t), x(t)\} \leqslant \frac{1}{n} \text { or } \max \{S(t), x(t)\} \geqslant n\right\}
$$

where throughout this paper, we set $\inf \varnothing=\infty$ (as usual, $\varnothing$ denotes the empty set). Clearly, $\tau_{n}$ is increasing as $n \rightarrow \infty$. Set $\tau_{\infty}=\lim _{n \rightarrow \infty} \tau_{n}$, whence $\tau_{\infty} \leqslant \tau_{e}$ a.s. It is easy to show that $\tau_{\infty}=\infty$ a.s. implies $\tau_{e}=\infty$ a.s. and $(S(t), x(t)) \in \mathbb{R}_{+}^{2}$ a.s. for all $t \geqslant 0$. In other words, to complete the proof we only need to show that $\tau_{\infty}=\infty$ a.s.

If this statement is not true, there will exist a pair of constants $T>0$ and $\varepsilon \in(0,1)$ such that

$$
P\left\{\tau_{\infty} \leqslant T\right\}>\varepsilon
$$

Hence there is an integer $n_{1} \geqslant n_{0}$ such that

$$
P\left\{\tau_{n} \leqslant T\right\}>\varepsilon, \quad \forall n \geqslant n_{1} .
$$

In addition, the total biomass $N(t)=S(t)+x(t)$ of the model (1.2) satisfies the following equation

$$
\mathrm{dN}(\mathrm{t})=\mathrm{D}\left(\mathrm{S}^{0}-\mathrm{N}(\mathrm{t})\right) \mathrm{dt} .
$$

By a simple calculation, it is easy to know that for all $t<\tau_{e}$,

$$
\mathrm{N}(\mathrm{t}) \leqslant \max \left\{\mathrm{S}_{0}+\mathrm{x}_{0}, \mathrm{~S}^{0}\right\}:=\mathrm{C} .
$$

Define a function $V: \mathbb{R}_{+}^{2} \rightarrow \mathbb{R}_{+}$by

$$
\mathrm{V}(\mathrm{S}(\mathrm{t}), x(\mathrm{t}))=-\ln \frac{\mathrm{S}}{\mathrm{C}}-\ln \frac{\mathrm{x}}{\mathrm{C}} .
$$

Obviously, $\mathrm{V}$ is positive defined. By Itô's formula, we get

$$
\begin{aligned}
d V= & {\left[-\frac{S^{0} D}{S}+2 D+\frac{m(x-S)}{a+S+K S^{2}}+\frac{\sigma^{2}\left(S^{2}+x^{2}\right)}{2\left(a+S+K S^{2}\right)^{2}}\right] d t } \\
& +\frac{\sigma(x-S)}{a+S+K S^{2}} d B(t) \\
:= & L V d t+\frac{\sigma(x-S)}{a+S+K S^{2}} d B(t),
\end{aligned}
$$


where $L$ is the generating operator of system (1.2), and by using (2.2), we can obtain

$$
\begin{aligned}
L V & \leqslant 2 D+\frac{m(x-S)}{a+S+K S^{2}}+\frac{\sigma^{2}\left(S^{2}+x^{2}\right)}{2\left(a+S+K S^{2}\right)^{2}} \\
& \leqslant 2 D+\frac{m C}{a}+\frac{\sigma^{2} C^{2}}{a^{2}}:=C_{0} .
\end{aligned}
$$

Therefore

$$
\int_{0}^{\tau_{n} \wedge T} d V(S(t), x(t)) \leqslant \int_{0}^{\tau_{n} \wedge T} C_{0} d t+\int_{0}^{\tau_{n} \wedge T} \frac{\sigma(x-S)}{a+S+K S^{2}} d B(t) .
$$

Taking the expectation of both sides yields

$$
\operatorname{EV}\left(S\left(\tau_{n} \wedge T\right), x\left(\tau_{n} \wedge T\right)\right) \leqslant V\left(S_{0}, x_{0}\right)+C_{0} T
$$

Set $\Omega_{n}=\tau_{n} \leqslant T$ for all $n \leqslant n_{1}$, due to (2.1), we know $P\left(\Omega_{n}\right) \geqslant \varepsilon$. Note that for every $\omega \in \Omega_{n}$, there is at least one of $S\left(\tau_{n}, \omega\right), x\left(\tau_{n}, \omega\right)$ equals either $n$ or $\frac{1}{n}$, and then

$$
\mathrm{V}\left(\mathrm{S}\left(\tau_{\mathrm{n}} \wedge \mathrm{T}\right), x\left(\tau_{\mathrm{n}} \wedge \mathrm{T}\right)\right) \geqslant\left(-\ln \frac{1}{\mathrm{nC}}\right) \wedge\left(-\ln \frac{\mathrm{n}}{\mathrm{C}}\right) .
$$

It follows from (2.3) and (2.1) that

$$
\begin{aligned}
\mathrm{V}\left(\mathrm{S}\left(\tau_{0}\right), x\left(\tau_{0}\right)\right)+\mathrm{C}_{0} \mathrm{~T} & \geqslant \mathrm{E}\left[\mathrm{I}_{\Omega_{\mathrm{n}}}\left(\mathrm{V}\left(\mathrm{S}\left(\tau_{\mathrm{n}}\right), x\left(\tau_{\mathrm{n}}\right)\right)\right)\right] \\
& \geqslant \varepsilon\left[\left(-\ln \frac{1}{\mathrm{nC}}\right) \wedge\left(-\ln \frac{\mathrm{n}}{\mathrm{C}}\right)\right],
\end{aligned}
$$

where $I_{\Omega_{n}}$ is the indicator function of $\Omega_{n}$. Letting $n \rightarrow \infty$ leads to the contradiction

$$
\infty>\mathrm{V}\left(\mathrm{S}_{0}, \mathrm{x}_{0}\right)+\mathrm{C}_{0} \mathrm{~T}=\infty .
$$

Thus we must have $\tau_{\infty}=\infty$ a.s. This completes the proof of Theorem 2.1.

Remark 2.2. If $\mathrm{m} \leqslant \mathrm{D}$, the microorganism must be washed out in chemostat, then we always assume $\mathrm{m}>\mathrm{D}$ in this paper.

\section{Extinction}

In this section, we will discuss the extinction for $x(t)$. The following lemma is useful for our proof which is a result in $[5,20]$.

Consider the one-dimensional time-homogeneous stochastic differential equation:

$$
d X=b(X) d t+\alpha(X) d B(t) \quad \text { with } X(0) \in \mathbb{R}_{+},
$$

satisfying the following conditions:

(1) $\alpha^{2}(X)>0$ for any $X \in I=(l, r)$ where $-\infty \leqslant l<r \leqslant \infty$;

(2) for any $X \in I$, there exists $\varepsilon>0$ such that $\int_{X-\varepsilon}^{X+\varepsilon} \frac{1+|b(X)|}{\alpha^{2}(X)} d x<\infty$.

Lemma 3.1 (See $[5,20])$. Assume that (1) and (2) hold. Let $\mathrm{X}(\mathrm{t})$ be a weak solution of (3.1) in $(\mathrm{l}, \mathrm{r})$. For some fixed constant $\mathrm{c} \in \mathrm{I}$, the scale function is defined as

$$
\mathrm{q}(x)=\int_{c}^{x} e^{-\int_{c}^{v} \frac{2 b(u)}{\alpha^{2}(u)} d u} d v
$$

If $\mathrm{q}\left(\mathrm{l}^{+}\right)>-\infty$ and $\mathrm{q}\left(\mathrm{r}^{-}\right)=\infty$ hold, then $\mathrm{P}\left(\lim _{\mathrm{t} \rightarrow \infty} \mathrm{X}(\mathrm{t})=\mathrm{l}\right)=\mathrm{P}\left(\sup _{\mathrm{t} \geqslant 0} \mathrm{X}(\mathrm{t})<\mathrm{r}\right)=1$. 
Theorem 3.2. If $R_{0}^{S}:=\frac{m S^{0}}{a+S^{0}+K\left(S^{0}\right)^{2}}-\frac{\sigma^{2}\left(S^{0}\right)^{2}}{2\left[a+S^{0}+K\left(S^{0}\right)^{2}\right]^{2}}<D$, then for any initial value $X(0)=x_{0} \in\left(0, S^{0}\right)$, the solution of $(1.3), x(t)$, obeys

$$
P\left(\lim _{t \rightarrow \infty} x(t)=0\right)=1,
$$

that is, the microorganism will be extinct with probability 1.

Proof. Note

$$
\begin{aligned}
& b(x)=\left[\frac{m\left(S^{0}-x\right)}{a+\left(S^{0}-x\right)+K\left(S^{0}-x\right)^{2}}-D\right] x, \\
& \alpha(x)=\frac{\sigma\left(S^{0}-x\right) x}{a+\left(S^{0}-x\right)+K\left(S^{0}-x\right)^{2}}, \quad c \in\left(0, S^{0}\right) .
\end{aligned}
$$

Compute that

$$
\begin{aligned}
\int_{c}^{x} \frac{2 b(u)}{\alpha^{2}(u)} d u= & \int_{c}^{x} 2 \cdot\left[\frac{m\left(S^{0}-u\right)}{a+\left(S^{0}-u\right)+K\left(S^{0}-u\right)^{2}}-D\right] u \\
& \cdot \frac{\left[a+\left(S^{0}-x\right)+K\left(S^{0}-x\right)^{2}\right]^{2}}{\sigma^{2}\left(S^{0}-u\right)^{2} u^{2}} d u \\
= & \frac{2}{\sigma^{2}} \int_{c}^{x}\left\{\left[2 D K^{2} S^{0}-K(m-2 D)\right]-D K^{2} \cdot u\right. \\
& +\frac{m S^{0}\left(a+S^{0}+K\left(S^{0}\right)^{2}\right)-D\left(a+S^{0}+K\left(S^{0}\right)^{2}\right)^{2}}{\left(S^{0}\right)^{2}} \cdot \frac{1}{u} \\
& +\frac{a m S^{0}-2 a D S^{0}-a^{2} D}{\left(S^{0}\right)^{2}} \cdot \frac{1}{S^{0}-u} \\
& \left.-\frac{a^{2} D}{S^{0}} \cdot \frac{1}{\left(S^{0}-u\right)^{2}}\right\} d u \\
= & -\frac{2}{\sigma^{2}}\left\{\frac{a m S^{0}-2 a D S^{0}-a^{2} D}{\left(S^{0}\right)^{2}} \ln \left(S^{0}-x\right)+\frac{a^{2} D}{S^{0}\left(S^{0}-x\right)}\right. \\
& -\frac{m S^{0}\left(a+S^{0}+K\left(S^{0}\right)^{2}\right)-D\left(a+S^{0}+K\left(S^{0}\right)^{2}\right)^{2}}{\left(S^{0}\right)^{2}} \ln x \\
& \left.-\left[2 D K^{2} S^{0}-K(m-2 D)\right] x+\frac{D K^{2}}{2} x^{2}\right\}+C_{0},
\end{aligned}
$$

where $C_{0}$ is a constant and clearly conditions (1) and (2) are satisfied. Then the scale function

$$
\begin{aligned}
\mathrm{q}(\mathrm{x}) & =\int_{c}^{x} e^{-\int_{\mathrm{c}}^{v} \frac{2 \mathrm{~b}(u)}{\alpha^{2}(u)} \mathrm{du}} d v \\
& =e^{-\mathrm{C}_{0}} \int_{c}^{x} e^{(A-B) v+E v^{2}} v^{F-G}\left(S^{0}-v\right)^{\mathrm{H}-\mathrm{I}} e^{\frac{\mathrm{J}}{\mathrm{S}^{0}-v}} \mathrm{~d} v,
\end{aligned}
$$

where $A=\frac{2 k m}{\sigma^{2}}, B=\frac{4 K D\left(K S^{0}+1\right)}{\sigma^{2}}, E=\frac{D K^{2}}{\sigma^{2}}, F=\frac{2 D\left(a+S^{0}+K\left(S^{0}\right)^{2}\right)^{2}}{\sigma^{2}\left(S^{0}\right)^{2}}, G=\frac{2 m S^{0}\left(a+S^{0}+K\left(S^{0}\right)^{2}\right)}{\sigma^{2}}, H=\frac{2 a m}{\sigma^{2} S^{0}}$, $\mathrm{I}=\frac{2 \mathrm{aD}\left(2 \mathrm{~S}^{0}+\mathrm{a}\right)}{\sigma^{2}\left(S^{0}\right)^{2}}, \mathrm{~J}=\frac{2 \mathrm{a}^{2} \mathrm{D}}{\sigma^{2} S^{0}}$ are all constants.

Let $w=\frac{1}{S^{0}-v}$ and $x \rightarrow\left(S^{0}\right)^{-}$, by (3.2)

$$
\begin{aligned}
\mathrm{q}\left(\left(S^{0}\right)^{-}\right) & \geqslant e^{-C_{0}} c^{F}\left(S^{0}\right)^{-G} e^{E c^{2}+A c-B S^{0}} \int_{c}^{S^{0}}\left(S^{0}-v\right)^{H-I} e^{\frac{J}{S^{0}-v}} d v \\
& \geqslant e^{-C_{0}} c^{F}\left(S^{0}\right)^{-G} e^{E c^{2}+A c-B S^{0}} \int_{\frac{1}{S^{0}-c}}^{+\infty} w^{I-H-2} e^{\frac{I w}{S^{0}}} d w \\
& \geqslant e^{-C_{0}} C_{1} \int_{\frac{1}{S^{0}-c}}^{+\infty} e^{\frac{I}{S^{0}} w} d w=\infty .
\end{aligned}
$$


Note that $R_{0}^{s}<D$ implies $F-G+1>0$, when $R_{0}^{s}<D$, let $x \rightarrow(0)^{+}$, we obtain

$$
\begin{aligned}
-\mathrm{q}\left((0)^{+}\right) & \leqslant e^{-\mathrm{C}_{0}}\left(S^{0}\right)^{\mathrm{H}}\left(\mathrm{S}^{0}-\mathrm{c}\right)^{-\mathrm{I}} e^{\frac{\mathrm{J}}{\mathrm{S}^{0}-\mathrm{c}}+A c+E c^{2}} \int_{0}^{c} v^{\mathrm{F}-\mathrm{G}} \mathrm{d} v \\
& \leqslant e^{-\mathrm{C}_{0}} \mathrm{C}_{2} \int_{0}^{\mathrm{c}} v^{\mathrm{F}-\mathrm{G}} \mathrm{d} v<\infty
\end{aligned}
$$

that is, $\mathrm{q}\left((0)^{+}\right)>-\infty$. By Lemma 3.1,

$$
P\left(\lim _{t \rightarrow+\infty} x(t)=0\right)=1 .
$$

The proof is complete.

\section{Persistence and stationary distribution}

In this section, we will talk about the persistence and stationary distribution for the microorganism $x(t)$.

Definition 4.1 (See [14]). The microorganism modeled by (1.3) is said to be

(1) persistence in mean, if $\lim _{t \rightarrow \infty} \frac{1}{t} \int_{0}^{t} x(s) d s \geqslant \zeta$ for some constant $\zeta>0$;

(2) stochastic persistence in the chemostat, if for any $\varepsilon \in(0,1)$, there exist positive constants $A_{1}=A_{1}(\varepsilon)$ and $A_{2}=A_{2}(\varepsilon)$ such that for any initial value $x_{0} \in \mathbb{R}_{+}$,

$$
\liminf _{t \rightarrow \infty} P\left(x(t) \leqslant A_{1}\right)>1-\varepsilon \text { and } \quad \liminf _{t \rightarrow \infty} P\left(x(t) \geqslant A_{2}\right)>1-\varepsilon .
$$

Lemma 4.2 (See [6]). Assume $\mathrm{b}$ and $\alpha$ the coefficients of (3.1) are twice continuously differentiable with second derivatives satisfying a Hölder condition, an invariant density exists if and only if the following two conditions hold

(1) $\int_{-\infty}^{x_{0}} \exp \left(-\int_{x_{0}}^{x} \frac{2 b(s)}{\alpha^{2}(s)} d s\right) d x=\int_{x_{0}}^{\infty} \exp \left(-\int_{x_{0}}^{x} \frac{2 b(s)}{\alpha^{2}(s)} d s\right) d x=\infty$;

(2) $\int_{-\infty}^{+\infty} \frac{1}{\alpha^{2}(x)} \exp \left(\int_{x_{0}}^{x} \frac{2 b(s)}{\alpha^{2}(s)} d s\right) d x<\infty$.

Furthermore, if an invariant density is twice continuously differentiable, then it satisfies the ordinary equation

$$
\mathrm{L}^{*} \pi=0, \text { that is } \frac{1}{2} \frac{\partial^{2}}{\partial \mathrm{y}^{2}}\left(\alpha^{2}(\mathrm{y}) \pi\right)-\frac{\partial}{\partial y}(\mathrm{~b}(\mathrm{y}) \pi)=0 .
$$

The solution is given by

$$
\pi(x)=\frac{C}{\alpha^{2}(x)} \exp \left(\int_{x_{0}}^{x} \frac{2 b(y)}{\alpha^{2}(y)} d y\right)
$$

where $C$ is found from $\int \pi(x) \mathrm{d} x=1$.

Theorem 4.3. Let $x(t)$ be the solution of system (1.3) with initial value $x_{0} \in\left(0, S^{0}\right)$. If $R_{0}^{s}>\mathrm{D}$, the microorganism $\mathrm{x}$ is stochastically persistent in the chemostat. The model (1.3) has a stationary distribution, denoted by $\pi(\mathrm{x})$.

Proof. Note

$$
\begin{aligned}
& b(x)=\left[\frac{m\left(S^{0}-x\right)}{a+\left(S^{0}-x\right)+K\left(S^{0}-x\right)^{2}}-D\right] x, \\
& \alpha(x)=\frac{\sigma\left(S^{0}-x\right) x}{a+\left(S^{0}-x\right)+K\left(S^{0}-x\right)^{2}}, \quad c \in\left(0, S^{0}\right) .
\end{aligned}
$$


Let $w=\frac{1}{S^{0}-c}$, compute that

$$
\begin{aligned}
\int_{0}^{S^{0}} \frac{1}{\alpha^{2}(v)} e^{\int_{c}^{v} \frac{2 b(u)}{\alpha^{2}(u)} d u d} d= & \frac{e^{C_{0}}}{\sigma^{2}}\left\{\int_{0}^{c}\left[a+S^{0}-v+K\left(S^{0}-v\right)^{2}\right]^{2}\right. \\
& \cdot e^{(B-A) v-E v^{2}-\frac{1}{S^{0}-v}} v^{G-F-2}\left(S^{0}-v\right)^{I-H-2} d v \\
& +\int_{c}^{S^{0}}\left[a+S^{0}-v+K\left(S^{0}-v\right)^{2}\right]^{2} \\
& \cdot e^{\left.(B-A) v-E v^{2}-\frac{1}{S^{0}-v} v^{G-F-2}\left(S^{0}-v\right)^{I-H-2} d v\right\}} \\
\leqslant & C_{3} \int_{0}^{c} v^{G-F-2} d v+C_{4} \int_{\frac{1}{S^{0}-c}}^{+\infty} e^{-J w} d w
\end{aligned}
$$

where $\mathrm{C}_{0}, \mathrm{C}_{3}, \mathrm{C}_{4}$ are all constants.

Under the condition $R_{0}^{s}>D$, we have

$$
\int_{0}^{S^{0}} \frac{1}{\alpha^{2}(v)} e^{\int_{c}^{v} \frac{2 b(u)}{\alpha^{2}(u)} d u} d v<\infty
$$

The conditions of Lemma 4.2 follow clearly from (3.3) and (4.1). Therefore, the microorganism $x$ is stochastically persistent in the chemostat. And the system (1.3) has a stationary distribution. In addition, the invariant density is given by

$$
\begin{aligned}
\pi(x)= & C\left[a+S^{0}-x+K\left(S^{0}-x\right)^{2}\right]^{2} \\
& \cdot e^{(B-A) x-E x^{2}-\frac{1}{S^{0}-x} x^{G-F-2}\left(S^{0}-x\right)^{I-H-2},}
\end{aligned}
$$

where $C$ is a constant such that $\int_{0}^{S^{0}} \pi(x) d x=1$.

Remark 4.4. If $\sigma \rightarrow 0, \mathrm{R}_{0}^{\mathrm{s}} \rightarrow \mathrm{R}_{0}$, then the properties of extinction and persistence for microorganism are consistent with the result of deterministic chemostat model in [2].

\section{Conclusion}

In this paper, we introduce the stochastic perturbation into a chemostat model with a Monod-Haldane response function. Firstly, we show that the system (1.3) has a unique global positive solution. Then through calculation, we obtain the threshold $R_{0}^{\mathrm{s}}$. Theorems 3.2 and 4.3 show that the microorganism will be extinct if $R_{0}^{s}<D$, and the continuous culture of the microorganism is successful if $R_{0}^{s}>D$. Thus we consider $R_{0}^{s}$ as the threshold of extinction and persistence for the microorganism. Theorem 4.3 also illustrates that the system (1.3) has a stationary distribution. Finally, we derive the expression for its invariant density.

\section{Acknowledgment}

The work was supported by National Natural Science Foundation of China (No. 11426113,1170012062), Project of Science and Technology Development Plan for Jilin Province (No. 20160520110JH), the key project of Jilin University of Finance and Economics (No. 0800091602).

\section{References}

[1] L. Arnold, Stochastic differential equations: theory and applications, Translated from the German, Wiley-Interscience [John Wiley \& Sons], New York-London-Sydney, (1972). 2

[2] L. S. Chen, J. Chen, Nonlinear biodynamical system, Science Press, Beijing, (1993). 1, 4.4

[3] D. Herbert, R. Elsworth, R. C. Telling, The continuous culture of bacteria; a theoretical and experimental study, Microbiology, 14 (1956), 601-622.1 
[4] T. W. James, Continuous culture of microorganisms, Ann. Rev. Microbiol., 15 (1961), 27-46. 1

[5] I. Karatzas, S. E. Shreve, Brownian motion and stochastic calculus, Graduate Texts in Mathematics, Springer-Verlag, New York, (1988). 3, 3.1

[6] A. Y. Kutoyants, Statistical inference for ergodic diffusion processes, Springer Series in Statistics, Springer-Verlag London, Ltd., London, (2004). 4.2

[7] Y.-G. Lin, D.-Q. Jiang, P.-Y. Xia, Long-time behavior of a stochastic SIR model, Appl. Math. Comput., 236 (2014), 1-9. 1

[8] M. Liu, C.-Z. Bai, Analysis of a stochastic tri-trophic food-chain model with harvesting, J. Math. Biol., 73 (2016), $597-625$.

[9] M. Liu, C.-Z. Bai, Optimal harvesting of a stochastic mutualism model with Léry jumps, Appl. Math. Comput., 276 (2016), 301-309.

[10] M. Liu, C.-Z. Bai, Optimal harvesting of a stochastic delay competitive model, Discrete Contin. Dyn. Syst. Ser. B, 22 (2017), 1493-1508.

[11] M. Liu, C.-Z. Bai, Y. Jin, Population dynamical behavior of a two-predator one-prey stochastic model with time delay, Discrete Contin. Dyn. Syst., 37 (2017), 2513-2538.

[12] M. Liu, M. Fan, Permanence of stochastic Lotka-Volterra systems, J. Nonlinear Sci., 27 (2017), 425-452.

[13] M. Liu, M. Fan, Stability in distribution of a three-species stochastic cascade predator-prey system with time delays, IMA J. Appl. Math., 82 (2017), 396-423. 1

[14] J.-L. Lv, K. Wang, Almost sure permanence of stochastic single species models, J. Math. Anal. Appl., 422 (2015), 675-683. 4.1

[15] X.-R. Mao, Stochastic differential equations and their applications, Horwood Publishing Series in Mathematics \& Applications, Horwood Publishing Limited, Chichester, (1997). 1, 2

[16] J. Monod, La technique de culture continue: theorie et applications, Annales de I Institut Pasteur, 79 (1950), 390-410. 1

[17] A. Novick, L. Szilard, Description of the chemostat, Science, 112 (1950), 715-716. 1

[18] B. Øksendal, Stochastic differential equations, An introduction with applications, Sixth edition, Universitext, Springer-Verlag, Berlin, (2003). 1

[19] C.-Q. Xu, S.-L. Yuan, An analogue of break-even concentration in a simple stochastic chemostat model, Appl. Math. Lett., 48 (2015), 62-68. 1

[20] Q.-S. Yang, D.-Q. Jiang, A note on asymptotic behaviors of stochastic population model with Allee effect, Appl. Math. Model., 35 (2011), 4611-4619. 3, 3.1 